\title{
Simplified-antiresonant reflecting optical waveguide-type vertical-cavity surface-emitting lasers
}

\author{
D. Zhou and L. J. Mawst ${ }^{\text {a) }}$ \\ Reed Center for Photonics, University of Wisconsin-Madison, Madison, Wisconsin 53706
}

(Received 7 December 1999; accepted for publication 4 February 2000)

\begin{abstract}
A two-step metalorganic chemical vapor deposition growth process is used to fabricate antiguided vertical-cavity surface-emitting lasers (VCSELs) incorporating a simplified-antiresonant reflecting optical waveguide (S-ARROW) design. Preliminary results show single-mode cw operation up to 1 $\mathrm{mW}$ output power from a $12 \mu \mathrm{m}$-diam $(\lambda=930 \mathrm{~nm})$ S-ARROW VCSEL with a large lateral index step $(\Delta n=0.1)$. Modal discrimination in the S-ARROW-VCSEL is calculated using a fiber-mode approximation and device optimization for high-single-mode powers is discussed. (C) 2000 American Institute of Physics. [S0003-6951(00)05013-0]
\end{abstract}

Vertical-cavity surface-emitting lasers (VCSELs) have been of great interest due to their low-threshold, high fiber coupling efficiency, and compact size that make it easier to integrate. Single-mode VCSELs with output powers in the $5-20 \mathrm{~mW}$ range would be especially useful for application such as laser printing $(\lambda=0.780 \mu \mathrm{m})$ and telecommunications $(\lambda=1.3 \mu \mathrm{m})$. Promising results in the $3-5 \mathrm{~mW}$ range $(\lambda=0.85 \mu \mathrm{m})$ have been obtained from wet-oxidized VCSELs. ${ }^{1,2}$ However, due to their weak (positive-) lateral index-guiding nature, they are very susceptible to gain spatial hole burning and thermal waveguiding, making the VCSEL aperture for single-mode operation very limited in size. To date, the highest fundamental-mode $\mathrm{cw}$ output power is $4.8 \mathrm{~mW}$ from a 3.5 - $\mu \mathrm{m}$-diam oxidized VCSEL, achieved by placing the oxide aperture at the optical field standing-wave null position. ${ }^{2}$

To obtain higher single-mode powers, the use of a negative-index guide (antiguide) is beneficial. Antiguides have demonstrated high-power, single-mode operation, from edge-emitting lasers ${ }^{3}$ and, more recently, have been implemented in VCSELs. ${ }^{4-6}$ The advantage of an antiguide structure is that it provides strong lateral radiation losses, which are highly mode dependent, thus filtering out higher-order spatial modes (even for large diameter devices, $d>6 \mu \mathrm{m})$. In addition, a large index-step $(\Delta n>0.05)$ provides for mode stability against carrier- and thermally induced index variations. Antiguided VCSELs have been fabricated either by surrounding a low index core region by regrowth of a highindex material ${ }^{4}$ or by creating a low-index core region by shifting the cavity resonance (towards longer wavelength) outside the core. ${ }^{5,6}$ The latter structure relies on the cavityinduced index step proposed by Hadely. ${ }^{7}$ These devices display promising results; single-mode operation up to 5-15 $\times I_{\text {th }}$ for diameters as large as $16 \mu \mathrm{m}$ wide have been achieved. ${ }^{4-6}$ On the other hand, the power has been limited to $<2 \mathrm{~mW}$ because of the relatively large radiation loss incurred for the fundamental mode, which is inherent to the antiguide structure. ${ }^{8}$

In order to reduce the edge radiation losses for the fun-

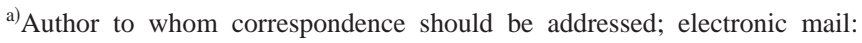
mawst@engr.wisc.edu
}

damental mode of an antiguide structure, antiresonant reflecting optical waveguides (ARROWs) have been employed for lateral waveguiding in edge emitting lasers. ${ }^{9}$ In the ARROW structure, a low-index core region is surrounded by a pair of quarter-lateral wave reflector regions, which are designed to be antiresonant for the fundamental mode. Higher-order modes, which do not meet the antiresonant condition, suffer large lateral radiation losses and are suppressed. In fact, improved modal discrimination, compared with a single antiguide, favoring the fundamental mode is created. This permits a large built-in lateral index step with a relatively large spot size $(>10 \mu \mathrm{m}$ diameter), suitable for high-power single-mode operation. A simplified version of the ARROW structure (S-ARROW), which is designed by taking out the low-index outer-reflector regions, has been found theoretically to be less sensitive to gain-spatial-hole burning than the conventional ARROW structure. ${ }^{10}$ Here, we propose and demonstrate preliminary results of an S-ARROW-type VCSEL ${ }^{11}$ as a means to reduce the antiguiding losses for the fundamental mode, allowing potential for

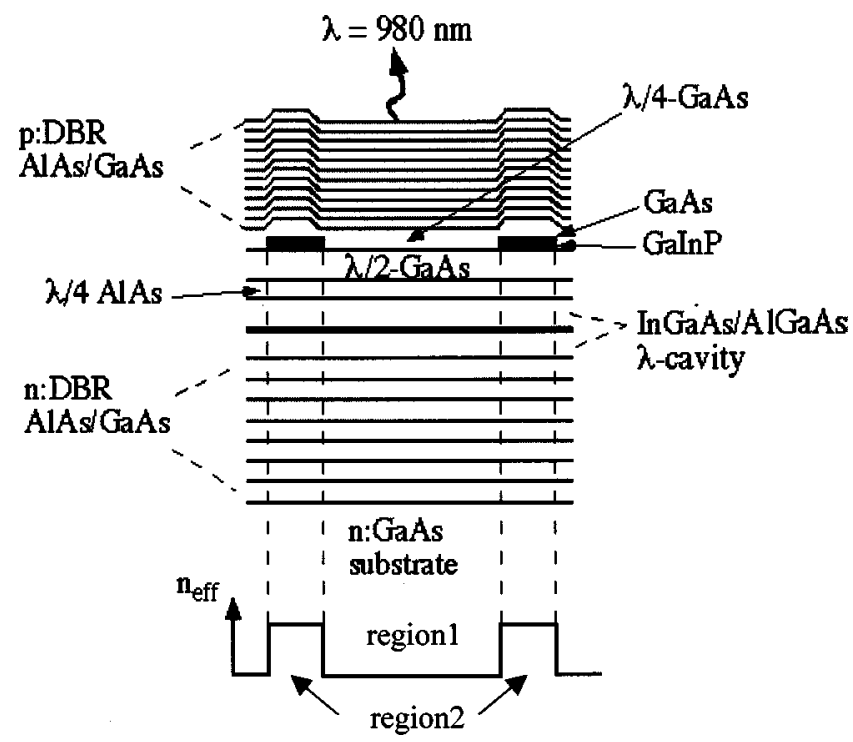

FIG. 1. Schematic structure of $980 \mathrm{~nm}$ S-ARROW VCSEL. The two additional layers of GaAs and GaInP provide the lateral variation of effective refractive index. 


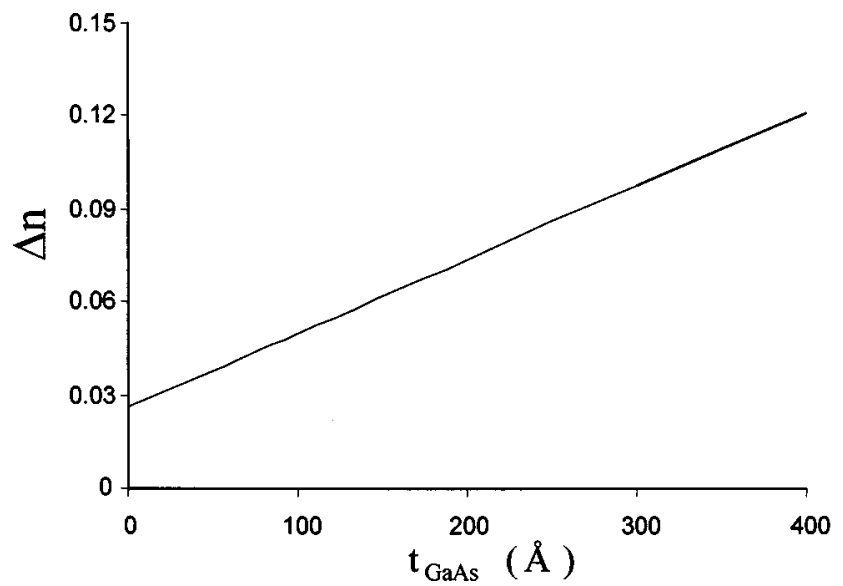

FIG. 2. Effective refractive index step as a function of the thickness of the GaAs spacer layer for the $980 \mathrm{~nm}$ VCSEL, shown in Fig. 1, with $t_{\text {GaInP }}$ $=12 \mathrm{~nm}$.

single-mode operation to high output powers. A schematic cross section of the $980 \mathrm{~nm}$ VCSEL incorporating an S-ARROW structure is shown in Fig. 1, along with the lateral effective refractive index profile.

The lateral waveguide is created by locally shifting the cavity resonance toward longer wavelength in a ring surrounding the emitting aperture (region 2 in Fig. 1). Hadley ${ }^{7}$ has shown that an increase in the resonant wavelength is equivalent to an increase of effective index according to the approximate relation; $\Delta n / n=\Delta \lambda / \lambda$. Figure 2 shows the calculated effective-index step between the low-index core region (region 1 in Fig. 1) and the surrounding high-index reflector ring region (region 2 in Fig. 1) for the S-ARROW VCSEL structure, as a function of the thickness of the GaAs spacer layer (the underlying GaInP layer thickness is fixed at $12 \mathrm{~nm}$ ). In our structure, the lateral index step $\Delta n$ is designed to be in the range $0.05-0.1$, corresponding to a GaAs thickness of 11-33 nm (see Fig. 2), which is a sufficiently large value to ensure mode stability. For a given $\Delta n$, a onedimensional (1D) model, can be used to estimate the width of the reflecting ring $s$, which will satisfy the antiresonance condition; $s=m \lambda_{1} / 4$, where $m$ is an odd integer and $\lambda_{1}$ is the lateral wavelength in the high-index region. ${ }^{9}$ In this work we have targeted devices with a reflector width of $2 \mu \mathrm{m}$, corresponding to $\sim 5 / 4 \lambda_{1}$. Further optimization to achieve the antiresonance condition will require an accurate 3D VCSEL waveguide calculation.

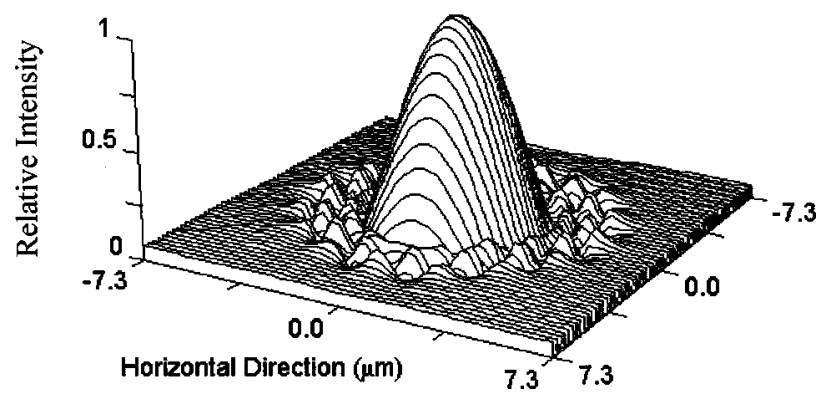

FIG. 3. Calculated fundamental mode intensity profile of a 6 - $\mu$ m-diam S-ARROW VCSEL for an effective refractive index step of 0.05 . The reflector regions correspond to an optical thickness of $5 / 4 \lambda_{1}$, where $\lambda_{1}$ is the

lateral wavelength of the radiation leakage.
Downloaded 04 Jan 2007 to 128.104.30.229. Redistribution subject to AIP license or copyright, see http://apl.aip.org/aper

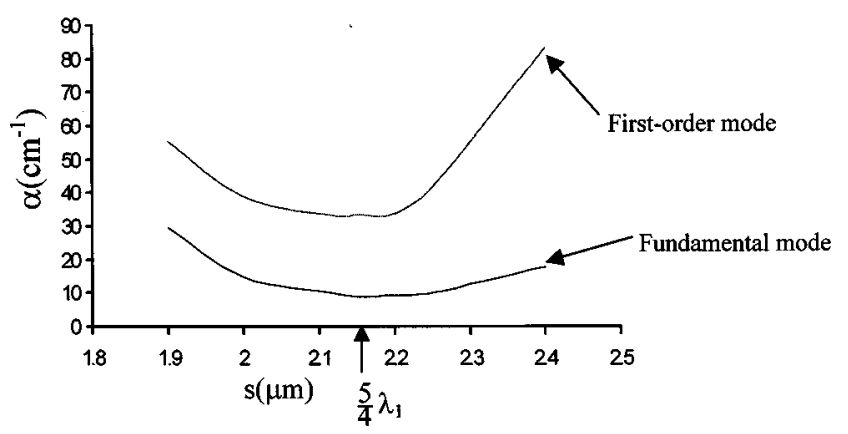

FIG. 4. Calculated modal loss as the function of reflector width, the antiresonant point which corresponds to $5 / 4 \lambda_{1}$, shows a low fundamental-mode loss and high modal discrimination.

As an approximation to an exact 3D VCSEL simulation, the lowest order modes for ( $6 \mu \mathrm{m}$ diameter, $\Delta n=0.05)$ of the S-ARROW VCSEL have been simulated (see Fig. 3) using an effective index model and fiber-mode approximation. As expected, the modal loss for the fundamental mode is significantly reduced ( $9 \mathrm{vs} 47 \mathrm{~cm}^{-1}$ ) compared with that of a single antiguide of the same diameter and index step. Furthermore, since the higher-order mode loss remains large in the S-ARROW structure, the ratio of the fundamental mode loss to the first-order mode loss is increased to $\sim 4$ (compared with $\sim 2.5$ for a single-antiguide structure). The modal loss as a function of the reflector width $s$ has been calculated as shown in Fig. 4. The antiresonance design point corresponds to the lowest loss region for the fundamental mode. In addition, a relatively large region exists around the antiresonance point where strong high-order mode discrimination occurs, with low loss for the fundamental mode. Away from antiresonance, higher mode discrimination is achieved at the expense of high fundamental mode loss. Similar behavior occurs for large index-step structure (i.e., $\Delta n=0.1$ ) although lower values of edge losses will occur.

The fabrication of the S-ARROW VCSEL involves a two-step low pressure MOCVD growth at $700{ }^{\circ} \mathrm{C}$. After the first growth, involving layers up to the $\operatorname{GaInP}(12 \mathrm{~nm})$ and GaAs(33 nm) spacers, we photoresist pattern the wafer and use selective chemical etching to form the ring reflector. The

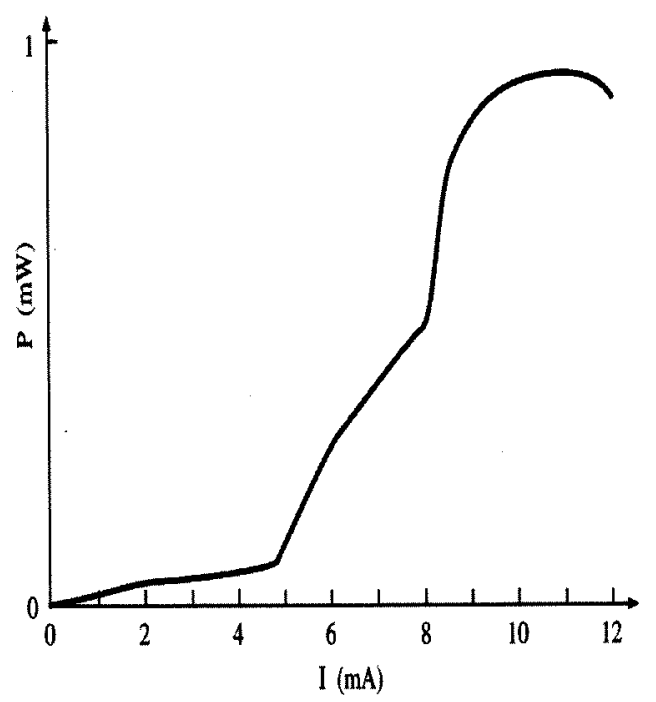

FIG. 5. Measured cw $P-I$ curve of a S-ARROW VCSEL with $12-\mu \mathrm{m}$-diam aperture. The thermal rollover limits the output power.

- 


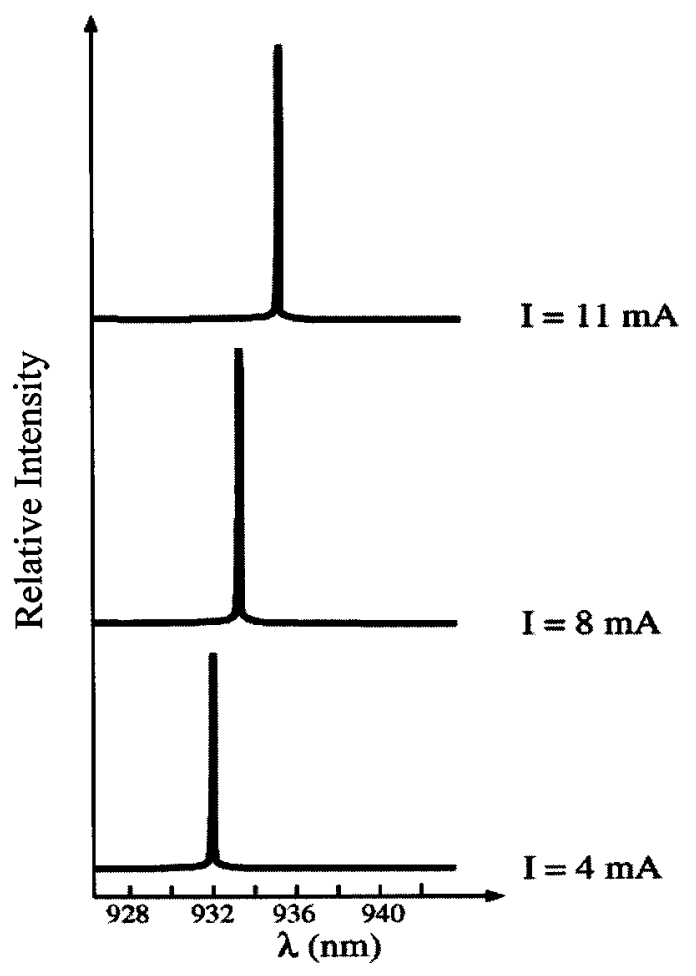

FIG. 6. Measured cw spectrum of a 12- $\mu$ m-diam S-ARROW device exhibits single-mode operation until thermal rollover at $1 \mathrm{~mW}$.

second growth involves the regrowth of the top $p$-type distributed Bragg reflector (DBR) and $p+\mathrm{GaAs}$ contact layer. Proton implantation is then used to confine the current to the central low-index core region. Finally, metal liftoff is used to open a window in the metal contact for light output.

Initial devices were fabricated with an index step $\Delta n$ $=0.05,{ }^{11}$ corresponding to a GaAs spacer thickness of 11 $\mathrm{nm}$. Near-field and spectral studies on these devices indicate lasing occurs first for the low-loss guided modes of the reflector ring. At higher injection currents, the leaky modes of the low-index core regions reach threshold. In order to achieve lasing only in the leaky modes of the structure, the antiguiding edge losses need to be reduced. To reduce the edge losses for the leaky modes, a higher index step $(\Delta n$ $=0.1$ ) design was implemented.

Preliminary results for devices with 12 - $\mu$ m-diam core regions and a larger index step of 0.1 are shown in Figs. 5 and 6. Threshold currents are $4.5 \mathrm{~mA}$ with single-mode operation (Fig. 6) up to $\sim 1 \mathrm{~mW} \mathrm{cw}$ output power until thermal rollover occurs at $\sim 3 \times I_{\text {th }}(12 \mathrm{~mA})$. A kink is observed in the power-current $(P-I)$ characteristics at $\sim 9 \mathrm{~mA}$, however, multimode operation does not occur, as evident from the spectral characteristics (Fig. 6) and far-field radiation pattern (full width half maximum $=10^{\circ}$ ). The kink and thermal rollover observed in the $P-I$ characteristics are believed to be a consequence of the strong device heating due to an unoptimized $p$-type DBR $\left(V_{\text {th }} \sim 4 \mathrm{~V}\right)$. In addition misalignment of the gain peak $(\lambda \sim 950 \mathrm{~nm})$ with the resonant wavelength $(\lambda \sim 930 \mathrm{~nm})$ also leads to premature thermal rollover. As a result further optimization of the voltage drop in the $p$-type DBR and alignment of the gain and cavity are expected to lead to a considerable increase in single-mode power. Finally, optimization of the lateral index step is required to ensure single-mode operation to high powers $(>10 \mathrm{~mW}$ from $12 \mu \mathrm{m}$ diameter).

In conclusion, we have demonstrated a simplified antiresonant reflecting optical waveguide-type (S-ARROW) VCSEL which has the potential for high power $(>10 \mathrm{~mW})$, single mode operation. Design curves indicate a large-index step device can be fabricated with low losses for the fundamental antiguided mode. Preliminary results are $1 \mathrm{~mW} \mathrm{cw}$ single-mode operation, up to three times threshold current, from a $12-\mu \mathrm{m}$-diam S-ARROW VCSEL.

The authors would like to acknowledge support from Agilent Technologies and NSF Career Award No. 9734283.

${ }^{1}$ K. D. Choquette, H. Q. Hou, G. R. Hadley, and K. M. Geib, 1997 Summer Topical Meeting on Vertical-Cavity Lasers, Montreal, Quebec, Canada, August 1997.

${ }^{2}$ C. Jung, R. Jager, M. Grabherr, P. Schnitzer, R. Michalzik, B. Weigl, S. Muller, and K. Jebeling, Electron. Lett. 33, 1790 (1997).

${ }^{3}$ D. Botez, L. J. Mawst, P. Hayashida, G. Peterson, and T. J. Roth, Appl. Phys. Lett. 53, 464 (1988).

${ }^{4}$ Y. A. Wu, G. S. Li, R. F. Nabiev, K. D. Choquette, C. Caneau, and C. J. Chang-Hasnain, IEEE J. Sel. Top. Quantum Electron. 1, 629 (1995).

${ }^{5}$ T. H. Oh, M. R. McDaniel, D. L. Huffaker, and D. G. Deppe, IEEE Photonics Technol. Lett. 10, 12 (1998).

${ }^{6}$ K. D. Choquette, G. R. Hadley, H. Q. Hou, K. M. Geib, and B. E. Hammons, Electron. Lett. 34, 991 (1998).

${ }^{7}$ G. R. Hadley, Opt. Lett. 20, 1483 (1995).

${ }^{8}$ R. W. Engelmann and D. Kerps, IEE Proc., Part I: Solid-State Electron Devices 127, 330 (1980).

${ }^{9}$ L. J. Mawst, D. Botez, C. Zmudzinski, and C. Tu, Appl. Phys. Lett. 61, 503 (1992).

${ }^{10}$ I. V. Goltser, L. J. Mawst, and D. Botez, Opt. Lett. 20, 2219 (1995).

${ }^{11}$ D. Zhou and L. J. Mawst, Conference Proceedings of IEEE LEOS 12th Annual Meeting, San Francisco, CA, November 1999, Proc. IEEE, Vol. 1, p. 393. 